\title{
Three-dimensional modeling and simulation of biceps femoris based on Matlab
}

\author{
Gang Tang ${ }^{1,2}$, Yanchao Huo ${ }^{1}$, Xiong Hu${ }^{1,}$ a, Ziyi Feng ${ }^{1}$, Chao Wang $^{1}$, Tianhao Tang $^{1}$ and Christophe \\ Claramunt $^{2}$ \\ ${ }^{1}$ Logistics Engineering College, Shanghai Maritime University, Shanghai 201306, China \\ ${ }^{2}$ The French Naval Academy Research Institute, Naval Academy, Brest 29240, France
}

\begin{abstract}
In order to study the properties of human muscle, it's necessary to construct a three-dimensional model. Biceps femoris has been taken as an example in this paper. Firstly, point cloud data of biceps femoris at different angles were extracted by IMAGEWARE software. Then we can get different polynomial functions correspond to different contraction states of biceps femoris in the Matlab environment. Finally, we calculate the pairwise polynomial parameters and get the correlation of the muscle shapes in different joint angle. The morphological changes of biceps femoris in different joint angle can give us more information about the properties of human muscle.
\end{abstract}

Keywords: biceps femoris; three-dimensional model; matlab; correlation analysis.

\section{Introduction}

Nowadays, musculoskeletal injuries are a common phenomenon. In addition to muscle damages in heavy manual labor, sports injuries also occupy a large proportion. Muscle injury, which is caused by a sharp contraction under the action of external force or excessive tensile force, is the most common form of sports injuries. According to the statistics of Beijing research institute of sports medicine, this kind of damage accounts for more than $25 \%$ in the incidence of various muscle damage.

The major parts of muscle injuries are triceps surae, muscles of leg anterolateral and hamstring muscles [1]. Therefore, the biomechanics analysis of muscle in the state of movement is significant to predict state of lower limb muscle strength, learn the distribution of the sports injuries and make sports reasonably and scientifically.

\section{Three-dimensional modeling based on Matlab}

This study based on five adult healthy male volunteers. MRI measurement experiment was done to collect the data of biceps femoris when lower limbs were under knee flexion condition. Form the test, we get the point cloud data of biceps femoris in different flexion angle.

Using IMAGEWARE software [2], Muscle parallel section of biceps femoris muscle in three different angles can be acquired by using parallel section method. Then the point cloud data were

${ }^{a}$ Corresponding author : xionghu@shmtu.edu.cn 
imported to Matlab for three-dimensional modeling by using plot3 function and writing assignment statements. In order to improve the quality of graphic display, grid statements were used to draw grid[3]. Three-dimensional curves in different angles of biceps femoris are shown in Fig. 1.

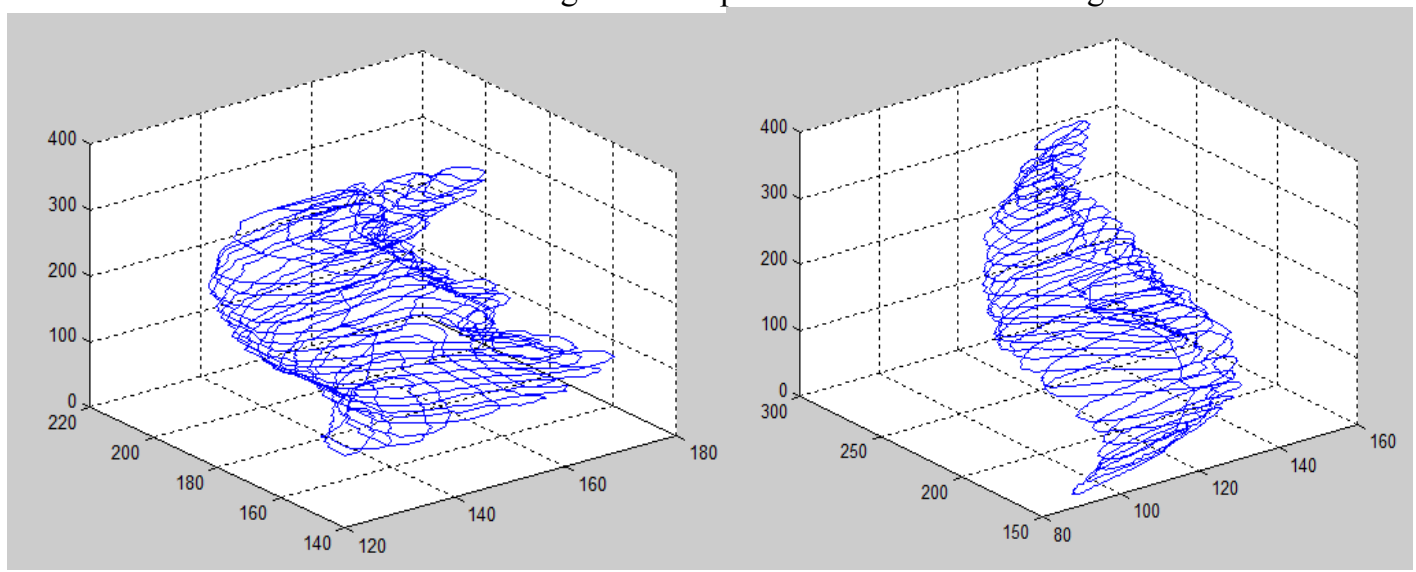

(a) $0^{\circ}$

(b) $30^{\circ}$

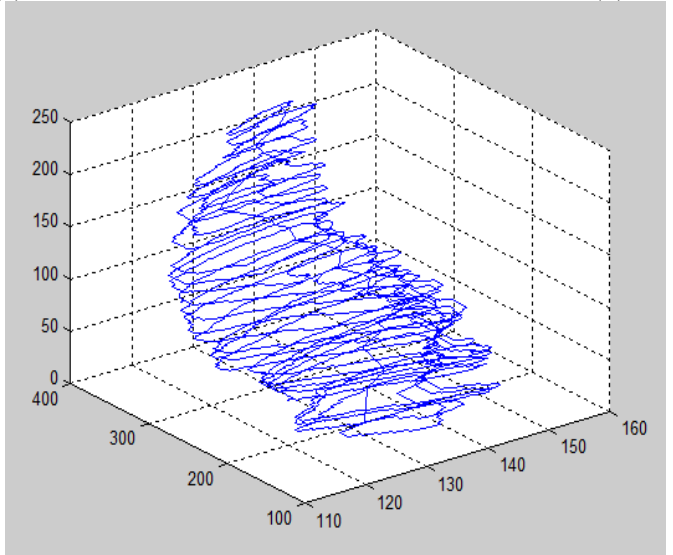

(c) $90^{\circ}$

Figure 1. Three-dimensional curves under different angles of the biceps femoris

Through analyzing the cross section of the parallel point cloud section, a similar cross graphic section always can be found in the same $\mathrm{Z}$ plane. For example, the similar curves of biceps femoris are shown in Fig. 2. 


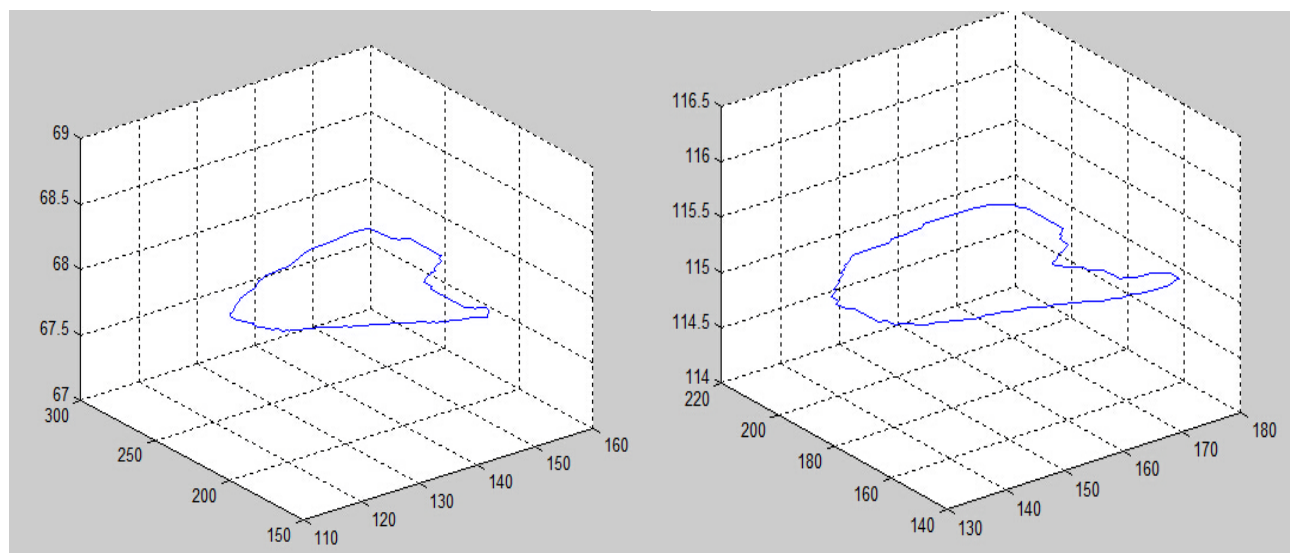

(a) $0^{\circ}$

(b) $30^{\circ}$

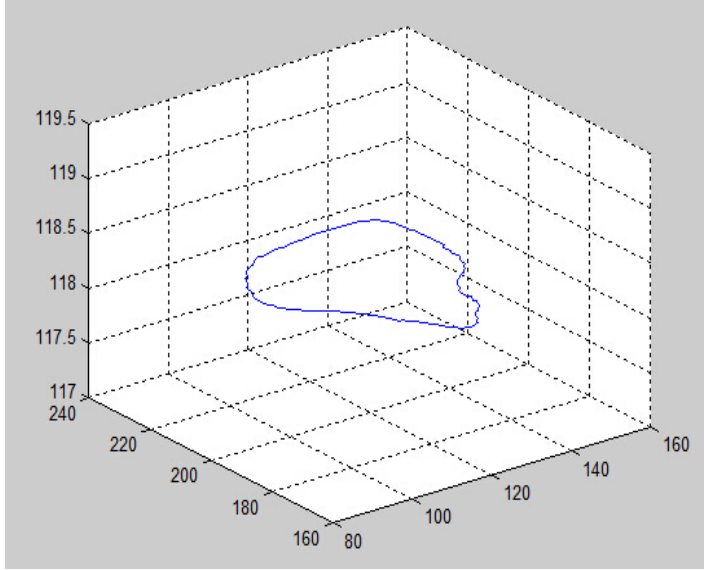

(c) $90^{\circ}$

Figure 2. Three-dimensional curves under different angles of the biceps femoris's single cross section

\section{Piecewise fitting of three-dimensional curves}

Because muscle curve shape is complicated, so it is difficult to use a function express. The purpose of the study is to find a general transformation matrix, which can get contraction state of different muscles. Because there is an error among transformation matrixes, so researchers usually use curve segments to reduce the error and enhance the correlation[4]. In this research, we transfer the three-dimensional curves of biceps femoris into two-dimensional curves in XOY plane. The curve segments are shown in Fig. 3. 


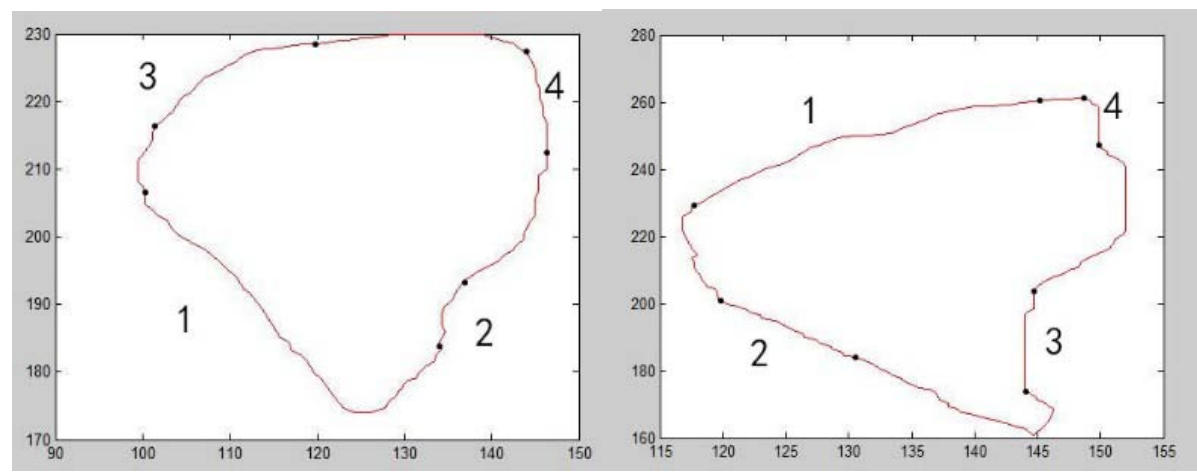

(a) $0^{\circ}$

(b) $30^{\circ}$

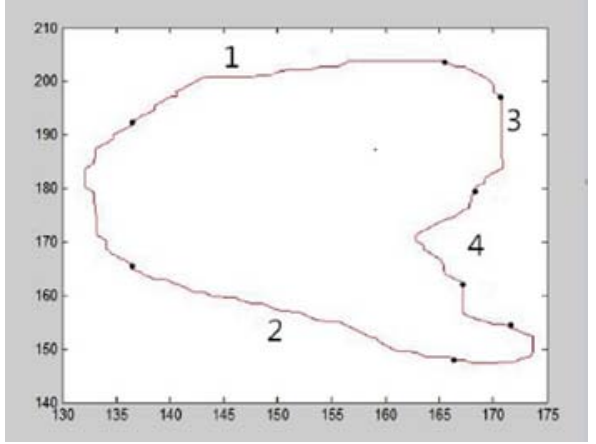

(c) $90^{\circ}$

Figure 3. Biceps femoris segmented curve under different angles of the biceps femoris

\section{The simulation results of the curves}

Through the simulation processes above, we get the segmented curves of biceps femoris in the angle of $0^{\circ}, 30^{\circ}$ and $90^{\circ}$, and each segmented curve has four parts. So we can get 24 thirteen-dimensional matrixes. Then choosing the second part of segmented curves in $0^{\circ}$, the first part of segmented curves in $30^{\circ}$ and $90^{\circ}$ as the example to make matrix transform. The matrix data are shown in Fig. 4.

$$
P_{1}=\mid \begin{aligned}
& 3.51913617902650 \mathrm{e}-16 \\
& 4.46500787545383 \mathrm{e}-13 \\
& 2.30340757353886 \mathrm{e}-10 \\
& -5.55777847186180 \mathrm{e}-08 \\
& 2.46419169800099 \mathrm{e}-06 \\
& 0.00217185568839754 \\
& -0.599462829964678 \\
& 57.7090590731439 \\
& 3163.07907531300 \\
& -1539581.63777394 \\
& 195358729.768081 \\
& -13210727805.4943 \\
& 484027151076.678
\end{aligned}
$$

(a) $0^{\circ}$

$P_{1}=\mid \begin{aligned} & 8.86286912934963 \mathrm{e}-16 \\ & -1.34924976661418 \mathrm{e}-12 \\ & 9.37769764435102 \mathrm{e}-10 \\ & -3.93783690078043 \mathrm{e}-07 \\ & 0.000111360292400346 \\ & -0.0223657800978418 \\ & 3.27554222877678 \\ & -353.140417839094 \\ & 27903.6671298073 \\ & -1584992.30003838 \\ & 62184366.5168785 \\ & -1561826271.01845 \\ & 21475527111.4819 \\ & -106101833601.928\end{aligned}$

(b) $30^{\circ}$

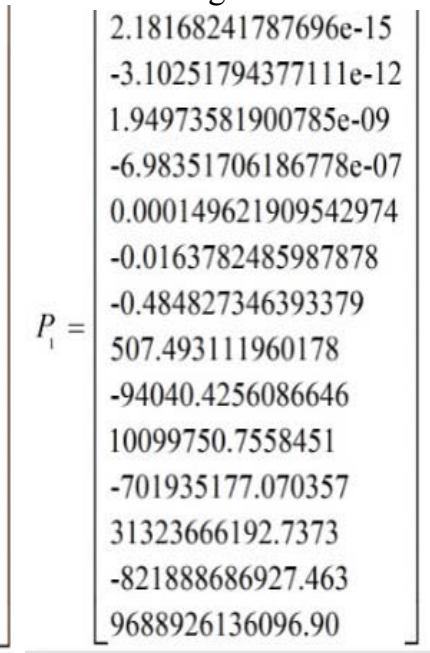

(c) $90^{\circ}$

Figure 4. Biceps femoris curve matrix 


\section{The correlation analysis of simulation data}

$\mathrm{A}$ is the transformation matrix between 0 degree and 30 degree of biceps femoris, and $\mathrm{B}$ is the transformation matrix between 30 degree and 90 degree of biceps femoris. Then we use SPSS software to analyze the two groups of data, the output is shown in Table 1[5].

Table 1 . Correlation analysis results of A

\begin{tabular}{|llll|}
\hline Correlation & & & \\
\hline \multirow{2}{*}{ VAR00001 } & Pearson correlation & VAR00001 & VAR00002 \\
& Significance (bilateral) & 1 & .423 \\
& Sum of cross product and square & 1127.614 & .0356 \\
& covariance & 178.845 & 28.793 \\
& $\mathrm{~N}$ & 7 & 5.166 \\
VAR00002 & Pearson correlation & .473 & 7 \\
& Significance (bilateral) & .364 & 1 \\
& Sum of cross product and square & 31.424 & \\
& covariance & 5.237 & .814 \\
& $\mathrm{~N}$ & 7 & 7 \\
\hline
\end{tabular}

From table 1, we can get the correlation coefficient $r=0.423$, which is between 0.3 to 0.5 , is low correlation. This kind of error appears because muscle cross section was uneven and the shape of each muscle is different. The two transform matrix were correlation, so the relationship of two pieces of muscle can be gotten in one transformation matrix.

\section{Conclusion}

In this paper, the three-dimensional model of biceps femoris were established and simulated by using the Matlab software. Through correlation analysis, the relative relationship of biceps femoris in different contraction angles were gotten. In the further research, we can get more experiment data, and analyse the relationship of the geometrical morphology between different muscles in different contraction state through combining the existing theories. This will give a hand to understand the rule of muscle movement deeply and treat muscle injuries effectively.

\section{Acknowledgements}

This work was supported by the National Natural Science Foundation of China (No. 31300783), China Postdoctoral Science Foundation (No. 2014M561458), Doctoral Fund of the Ministry of Education Jointly Funded Project (No. 20123121120004), the Shanghai Maritime University Research Project (No. 20130474), the Shanghai Top Academic Discipline Project- management science \& engineering, and the high-tech research and development program of China (No. 2013A2041106).

\section{References}

1. Garrett W E, Safran M R, Seaber A V, et al. Biomechanical comparison of stimulated and nonstimulated skeletal muscle pulled to failure [J]. The American journal of sports medicine, 1987, 15(5): 448-454.

2. ZHANG Y, Sheng L U, XU Y, et al. Application of navigation template to fixation of sacral fracture using three-dimensional reconstruction and reverse engineering technique[J]. Chinese Journal of Traumatology (English Edition), 2009, 12(4): 214-217. 
3. Pavlidis T. Algorithms for graphics and image processing[M]. Springer Science \& Business Media, 2012.

4. Motulsky H J, Ransnas L A. Fitting curves to data using nonlinear regression: a practical and nonmathematical review[J]. The FASEB journal, 1987, 1(5): 365-374.

5. Lorenzo-Seva U, Ferrando P J. FACTOR: A computer program to fit the exploratory factor analysis model[J]. Behavior research methods, 2006, 38(1): 88-91. 